\title{
Synthesis and characterization of stable aqueous dispersions of graphene
}

\author{
UJJAL KUMAR SUR ${ }^{1, *}$, ABHIJIT SAHA ${ }^{2}$, APARNA DATTA ${ }^{2}$, BALAPRASAD ANKAMWAR ${ }^{3}$, \\ FARAH SURTI ${ }^{3}$, SANNAK DUTTA ROY ${ }^{4}$ and DEBASISH ROY ${ }^{5}$ \\ ${ }^{1}$ Department of Chemistry, Behala College, Parnashree, University of Calcutta, Kolkata 700 060, India \\ ${ }^{2}$ UGC-DAE Consortium for Scientific Research, Kolkata Centre, III/LB-8 Bidhannagar, Kolkata 700 098, India \\ ${ }^{3}$ Bio-Inspired Materials Science Laboratory, Department of Chemistry, University of Pune, Ganeshkhind, \\ Pune 411 007, India \\ ${ }^{4}$ Department of Physics, Sammilani Mahavidyalaya, E.M. Bypass, Baghajatin Station, University of Calcutta, \\ Kolkata 700 075, India \\ ${ }^{5}$ Department of Zoology, Sammilani Mahavidyalaya, E.M. Bypass, Baghajatin Station, University of Calcutta, \\ Kolkata 700 075, India
}

MS received 11 August 2014; accepted 27 October 2014

\begin{abstract}
A stable aqueous dispersion $\left(5 \mathrm{mg} \mathrm{ml}^{-1}\right)$ of graphene was synthesized by a simple protocol based on three-step reduction of graphene oxide (GO) dispersion synthesized using the modified version of Hummers and Offeman method. Reduction of GO was carried out using sodium borohydride, hydrazine hydrate and dimethyl hydrazine as reducing agents. The chemically synthesized graphene was characterized by scanning electron microscopy (SEM), transmission electron microscopy (TEM), UV-visible absorption spectroscopy, Fourier transform infrared (FTIR) and Raman spectroscopy, thermogravimetric analysis (TGA), optical microscopy. The stability of aqueous dispersions of graphene was confirmed through zeta potential measurements and the negative zeta potentials of $55-60 \mathrm{mV}$ were obtained indicating the high stability of aqueous graphene dispersions.
\end{abstract}

Keywords. Graphene; zeta potential; graphene oxide; graphite; dispersion; Raman spectroscopy.

\section{Introduction}

Since the discovery of fullerene $\left(\mathrm{C}_{60}\right)$ in 1985 [1], several other carbon nanostructures have been isolated. In 1991, Japanese scientist Iijima [2] discovered carbon nanotube (CNT) while graphene, a two-dimensional form of graphite was isolated in 2004 by Geim and Novoselov of Manchester University, United Kingdom [3]. This new kind of carbon nanostructure has attracted tremendous attention both from fundamental and applied research point of view because of its high specific surface area, high chemical stability, high optical transmittance, high porosity, biocompatibility, tunable band gap and ease of chemical functionalization which actually helps in tuning its properties $[4,5]$. It can be stacked into three-dimensional (3D) graphite, wrapped up into zerodimensional (0D) fullerenes, rolled into one-dimensional (1D) nanotubes. The different protocols which have been reported in the literature up to now to synthesize graphene include micromechanical cleavage of graphite [3], chemical exfoliation of graphite [6], solvothermal synthesis [7], epitaxial growth on silicon carbide ( $\mathrm{SiC}$ ) surfaces and metal surfaces [8], chemical vapour deposition (CVD) of hydrocarbons on metal surfaces [9], bottom-up organic synthesis [10], and the reduction of graphene oxide (GO) obtained from graphite oxide by various reducing agents $[11,12]$.

*Author for correspondence (uksur99@yahoo.co.in)
The chemical reduction method is considered to be a scalable approach to obtain graphene owing to its simplicity and exceptionally low cost. This method has been used widely to synthesize chemically derived graphene. Graphite is first oxidized to graphite oxide using either the Hummers method [13] or the modified Hummers method [14] in the presence of strong acids and oxidizing agents. Graphite oxide can be readily exfoliated as individual GO sheets by ultrasonication in water. GO, an oxidized form of graphene is functionalized with hydroxyl $(-\mathrm{OH})$ and epoxy $(>\mathrm{O})$ functional groups on the hexagonal network of carbon atoms with carboxyl $(-\mathrm{COOH})$ and carbonyl $(-\mathrm{C}=\mathrm{O})$ groups at the edges. $\mathrm{GO}$ can be converted into graphene by chemical reduction process. The synthesized graphene sheets will lose their water dispersability and the graphene sheets aggregate and eventually precipitate. However, one can tune the water dispersability by introducing hydrophilic group into GO before it is fully reduced. The resulting graphene will create stable water dispersion without aggregation and precipitation.

Pristine graphene is insoluble in common polar solvents such as water. Therefore, it is necessary to attach certain functional groups on graphene either physically or chemically to disperse in various common solvents without significantly changing its desirable properties. This kind of functionalization alters the dispersability of graphene and ultimately provides fine control over its polarity. Therefore, functionalization of graphene is really a challenging task for scientist. 
A facile and scalable approach for the preparation of aqueous stable dispersions of sparingly sulphonated graphene sheets is reported here. In this method, graphite oxide is prepared first by oxidizing graphite flakes using concentrated sulphuric acid and sodium nitrite. This is followed by ultrasonication of graphite oxide in water to yield GO. Finally, graphene is prepared from GO in three steps. We have used hydrazine hydrate, dimethylhydrazine and sodium borohydride as reducing agents. The synthesized graphene sheets were characterized by scanning electron microscopy (SEM), transmission electron microscopy (TEM), UVvisible absorption spectroscopy, Fourier transform infrared (FTIR) and Raman spectroscopy, thermogravimetric analysis (TGA), optical microscopy. The stability of aqueous dispersions of graphene was confirmed through zeta potential measurements and the negative zeta potentials of $55-60 \mathrm{mV}$ were obtained.

\section{Experimental}

\subsection{Materials and characterization}

All the reagents/chemicals used for the synthesis of graphene such as graphite powder $(<20 \mu \mathrm{m}$, synthetic grade, SigmaAldrich), sulphuric acid 98\% (Merck, GR grade), potassium permanganate $>99 \%$ (Emerck), hydrogen peroxide 30\% (Merck), sodium carbonate pure (Merck), hydrochloric acid 35\% (Merck, GR grade), sodium nitrite extra pure (HIMEDIA company), sulphanilic acid 99\% (Aldrich, AR grade), sodium borohydride (Ranbaxy, AR grade), hydrazine hydrate 80\% (Rankem, AR grade), dimethylhydrazine 98\% (Aldrich) were used in highly purified form without any further purification in this study. Millipore water (MilliQ water) of resistivity $\sim 18.2 \mathrm{M} \Omega \mathrm{cm}$ was used for all aqueous solution preparation.

Scanning electron microscopic measurements were performed on a JEOL model JSM-6360A instrument operated at an accelerating voltage $20 \mathrm{kV}$. High-resolution transmission electron microscope (HR-TEM) (JEOL, model JEM-2010) instrument operated at an accelerating voltage at $120 \mathrm{kV}$ was used. Samples for TEM analysis were prepared by solution-casting the graphene samples on a carbon-coated TEM grid. Dynamic light scattering set-up (DLS; Model DLS-nano ZS, Zetasizer, Nanoseries, Malvern Instruments) was used for zeta potential measurement. The zeta potential was calculated from the electrophoretic mobility using the Smoluchowski equation with the help of commercial software installed in the instrument. UV-visible absorption spectroscopic studies were carried out on a JASCO V-630 spectrophotometer. TGA was performed on both GO and graphene samples in a Perkin Elmer (TGA7) from 50 to $700^{\circ} \mathrm{C}$ under nitrogen atmosphere (heating rate: $15^{\circ} \mathrm{C} \mathrm{min}^{-1}$ ) and the masses are recorded as a function of temperature. FTIR spectra of the samples were recorded on a Thermo Scientific NICOLET 6700 spectrophotometer using $\mathrm{KBr}$ pellets in the range of $4000-450 \mathrm{~cm}^{-1}$. Raman spectra of the as-prepared graphene samples were recorded on a LabRam I confocal microprobe Raman system (Horiba JobinYvon, France, Model no: HR 800) at $\lambda=514 \mathrm{~nm}$ laser power $1.7 \mathrm{~mW}$ as the excitation source. Optical microscope (Model no.: MLM Olympus) was used to take the optical micrographs of graphene sheets.

\subsection{Synthetic protocol for aqueous dispersion of graphene}

2.2a Synthesis of graphite oxide: Graphite oxide was synthesized from graphite powder by employing the modified version of Hummers and Offeman method. In this method, $23 \mathrm{ml}$ of $98 \%$ concentrated sulphuric acid $\left(\mathrm{H}_{2} \mathrm{SO}_{4}\right)$ was cooled down below $0{ }^{\circ} \mathrm{C}$ in an ice bath for 30-45 min. One gram of graphite powder and $0.5 \mathrm{~g}$ of sodium nitrite $\left(\mathrm{NaNO}_{2}\right)$ were mixed together and then added to cold concentrated $\mathrm{H}_{2} \mathrm{SO}_{4}$ with constant stirring in an ice bath for $45 \mathrm{~min}$. Three grams of potassium permanganate $\left(\mathrm{KMnO}_{4}\right)$ was slowly added to the above solution and temperature was maintained below $20^{\circ} \mathrm{C}$ with constant stirring for $15 \mathrm{~min}$ by keeping in the ice bath. The solution colour immediately turned greenish black from black, indicating the completion of oxidation. The solution was removed from the ice bath and allowed to attain the room temperature. Next, the solution was kept under oil bath at $60^{\circ} \mathrm{C}$ with constant stirring until the solution colour changes to brown. One-hundred and forty millilitres of deionized MilliQ water was added followed by the addition of $10 \mathrm{ml}$ of $30 \%$ hydrogen peroxide $\left(\mathrm{H}_{2} \mathrm{O}_{2}\right)$ solution to stop the reaction and kept on stirring for 15 min where dark brown colour was observed in the solution. The solution was kept to settle down for overnight and was centrifuged to obtain the product. The solid product was washed for several times with $5 \%$ of hydrochloric acid $(\mathrm{HCl})$ solution to remove impurities and sulphate ions. The product was then dried under vacuum using Rota evaporator at $50^{\circ} \mathrm{C}$ to obtain graphite oxide.

\section{$2.2 \mathrm{~b}$ Preparation of aqueous graphene dispersion from} graphite oxide: One hundred milligrams of the above prepared graphite oxide was dispersed in $100 \mathrm{ml}$ of MilliQ water followed by sonication for $1 \mathrm{~h}$. A clear brown coloured solution was obtained, which is the stable dispersion of GO in water. Subsequently, the $\mathrm{pH}$ of solution was adjusted up to 12 by adding $5 \%$ sodium carbonate solution. Graphene was prepared from GO in three steps. In the first step, GO was reduced with sodium borohydride solution $(750 \mathrm{mg}$ of $\mathrm{NaBH}_{4}$ in $20 \mathrm{ml}$ of MilliQ water) to remove the most of the oxygen functionality in GO. The aqueous mixture was kept under oil-bath at $80^{\circ} \mathrm{C}$ for $1 \mathrm{~h}$ with constant stirring. The aqueous mixture was centrifuged and washed repeatedly with MilliQ water. In the second step, the partially reduced GO was functionalized by carrying out sulphonation reaction with the aryl diazonium salt of sulphanilic acid. The aryl diazonium salt was obtained by mixing $50 \mathrm{mg}$ of sulphanilic acid and $20 \mathrm{mg}$ of sodium nitrite in $10 \mathrm{ml}$ of water with $0.5 \mathrm{ml}$ of $1 \mathrm{~N} \mathrm{HCl}$ solution at temperature below $0^{\circ} \mathrm{C}$ in ice bath. The partially reduced GO was again redispersed 
into $80 \mathrm{ml}$ of MilliQ water and sonicated for few minutes to get properly dispersed solution. The functionalization of the above dispersed solution of partially reduced GO was carried out in ice-bath with constant stirring for $3 \mathrm{~h}$. Again, the mixture was centrifuged and washed several times with MilliQ water to remove impurities and redispersed in $80 \mathrm{ml}$ of MilliQ water. In the third and last step, post-reduction was carried out to completely reduced sulphonated GO by removing any remaining oxygen functionality. Hydrazine hydrate and dimethylhydrazine were used as the reducing agents in this step. In total $4 \mathrm{~g}$ of hydrazine hydrate and $50 \mathrm{mg}$ of dimethyl hydrazine were mixed in $10 \mathrm{ml}$ of MilliQ water and the resulting solution was added to the dispersed solution and kept on stirring for $15 \mathrm{~h}$ at $100^{\circ} \mathrm{C}$. The obtained product was centrifuged and washed several times with MilliQ water and complete removal of sulphate ions were confirmed till we get clear solution without turbidity using $5 \mathrm{ml}$ of $5 \%$ sodium carbonate solution. The resulting final product can be readily dispersed in water at reasonable concentration $\left(5 \mathrm{mg} \mathrm{ml}^{-1}\right)$ in the $\mathrm{pH}$ range of $4-10$.

\section{Results and discussion}

\subsection{Characterization of as-prepared graphene samples}

The synthesized graphene sheets were characterized by SEM, TEM, UV-visible absorption spectroscopy, FTIR and Raman spectroscopy, TGA and optical microscopy.

Figure 1 shows the scanning electron microscopic image of the as-synthesized graphene. The image reveals flakes like structures of the samples. Figure 2 illustrates the TEM image (lower resolution) demonstrating the stacking of graphene sheets at lower resolution. It is evident from the TEM image that graphene flakes are stacked together and reveals as a multilayered structure. It shows that the particles are aggregated with a honeycomb structure and a very small particle size in the order of few tens of nanometre. The interpretation

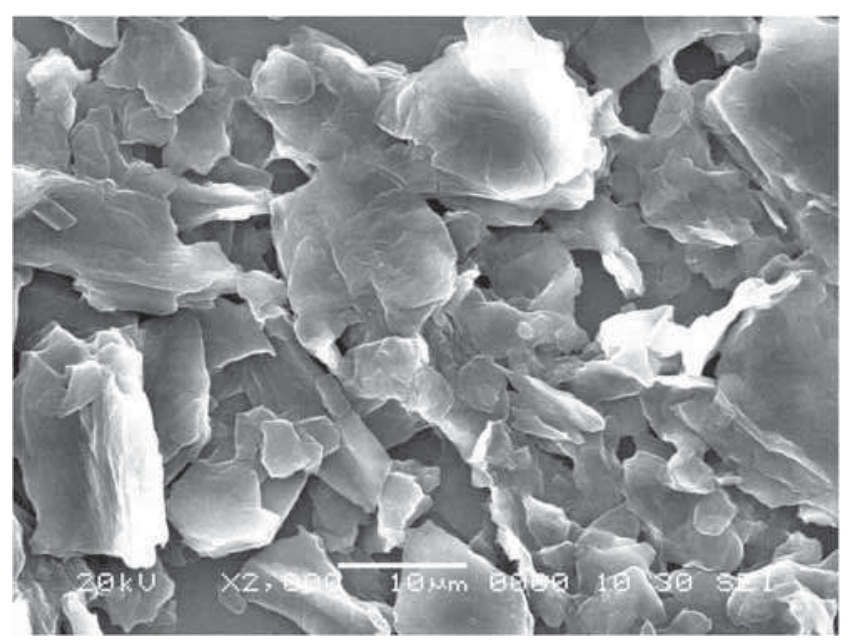

Figure 1. The scanning electron microscopic image of the as-synthesized graphene.

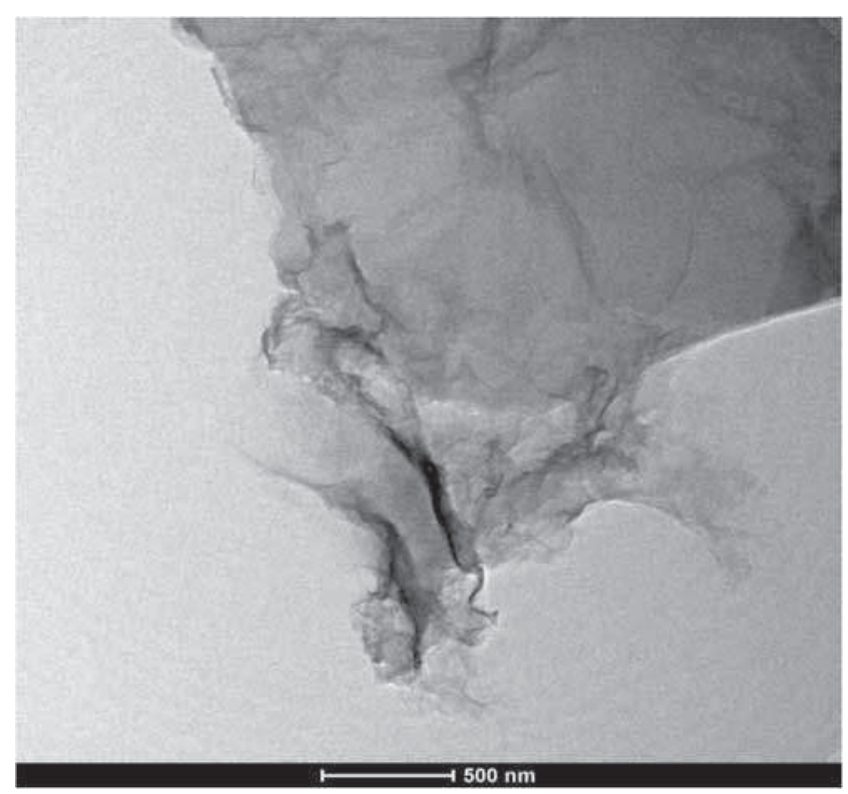

Figure 2. The transmission electron microscopic image (lower resolution) demonstrating the stacking of graphene sheets.

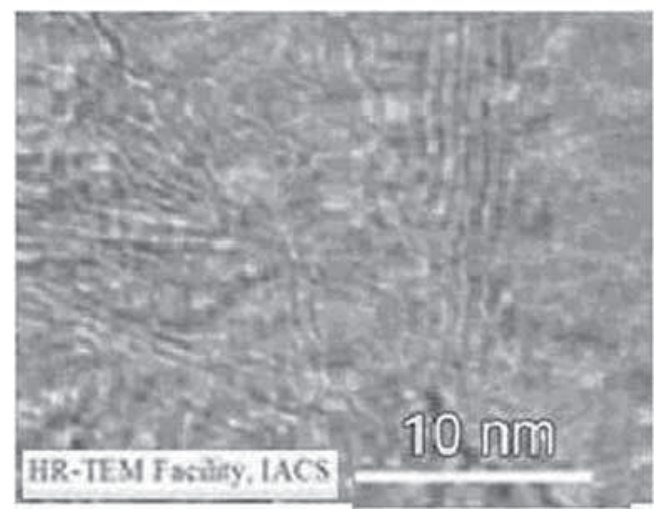

Figure 3. The HR-TEM image of graphene illustrating the crystal planes.

of particle size from the TEM images is difficult because of its irregular boundaries of the particles. The as-synthesized graphene exhibits typical wrinkled structure that caused sheet folding as demonstrated in the TEM images. Figure 3 shows the TEM image at higher resolution illustrating the crystal planes of graphene (HR-TEM).

Figure 4 shows the UV-visible absorption spectra of the $\mathrm{GO}$ as well as the synthesized graphene samples. The GO exhibits an absorption peak at $230 \mathrm{~nm}$ attributed to the $\pi-\pi^{*}$ transition of the aromatic $\mathrm{c}-\mathrm{c}$ ring and a weak broad absorption band (shoulder) around $300 \mathrm{~nm}$ corresponds to the $\mathrm{n}-\pi^{*}$ transition of $\mathrm{C}=\mathrm{O}$ bonds [15]. In the graphene samples, the absorption peak at $230 \mathrm{~nm}$ is red-shifted to $270 \mathrm{~nm}$, while the weak absorption at $300 \mathrm{~nm}$ is totally absent as illustrated in figure 4. The red-shift of the absorption peak in graphene can be explained by the decrease in oxygen functional groups and increase in aromatic rings (due to the reduction of GO 


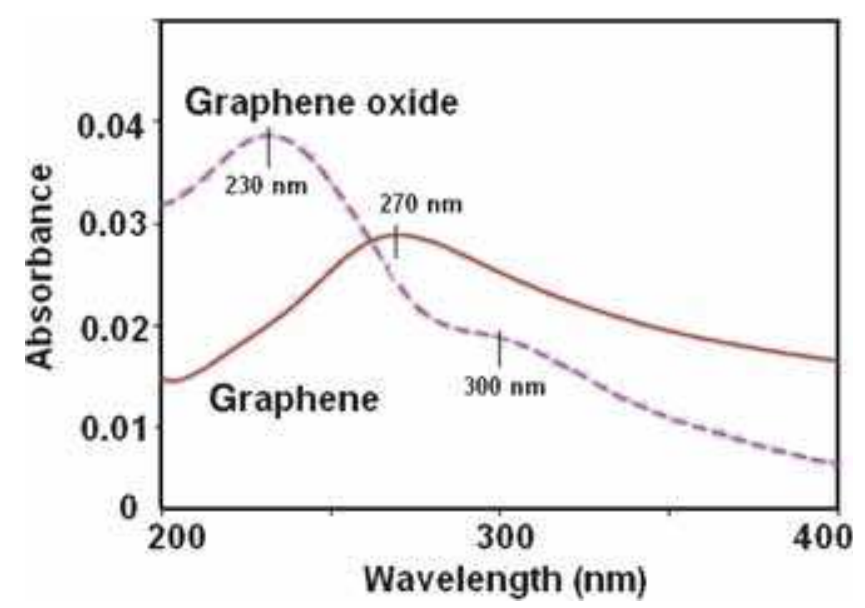

Figure 4. The UV-visible absorption spectra of the graphene oxide as well as the synthesized graphene samples.

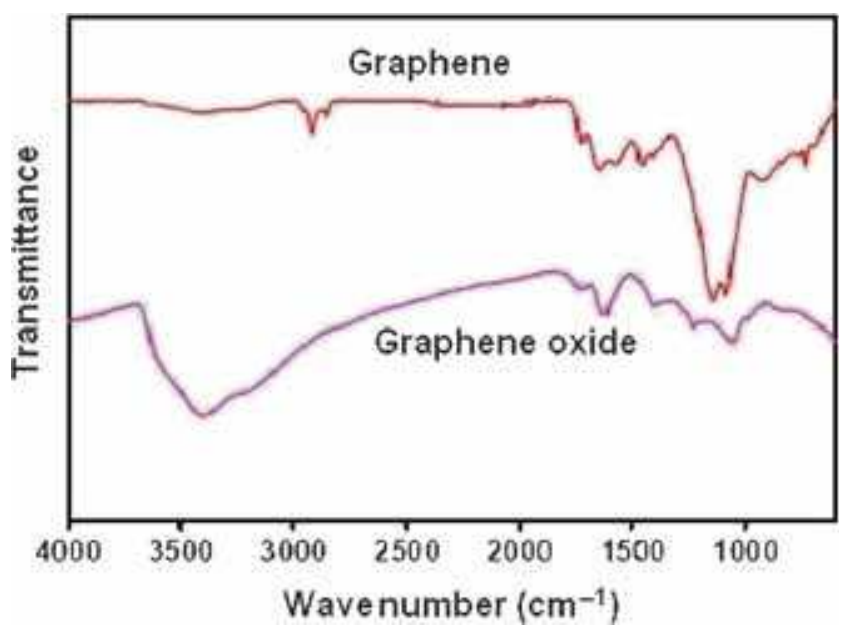

Figure 5. The FTIR spectra of graphene oxide and reduced graphene in the full range of wavenumbers $\left(450-4000 \mathrm{~cm}^{-1}\right)$.

into graphene) causing electrons to be excited easily at a lower energy (longer wavelength; red shift). The synthesized graphene exhibits an absorbance value of $\sim 0.03$, which corresponds to transmittance value of around $95 \%$. Therefore, graphene is optically transparent.

Figure 5 illustrates the FTIR spectra of GO and reduced graphene in the full range of wavenumbers $\left(450-4000 \mathrm{~cm}^{-1}\right)$. The different types of oxygen functionalities present in GO were confirmed by the FTIR spectra at $3400 \mathrm{~cm}^{-1}(\mathrm{O}-\mathrm{H}$ stretching vibrations), at $1720 \mathrm{~cm}^{-1}$ (stretching vibrations from $\mathrm{C}=\mathrm{O}$ ), at $1600 \mathrm{~cm}^{-1}$ (skeletal vibrations from unoxidized graphitic domains), at $1220 \mathrm{~cm}^{-1}$ (C-OH stretching vibrations) and at $1060 \mathrm{~cm}^{-1}$ (C-O stretching vibrations) [16,17]. In the FTIR spectra of as-synthesized graphene, the $\mathrm{O}-\mathrm{H}$ stretching vibrations observed at $3400 \mathrm{~cm}^{-1}$ was significantly reduced due to deoxygenation. However, stretching vibrations from $\mathrm{C}=\mathrm{O}$ at $1720 \mathrm{~cm}^{-1}$ were still observed and $\mathrm{C}-\mathrm{O}$ stretching vibrations at $1060 \mathrm{~cm}^{-1}$ became sharper. Figure 6 shows the FTIR spectra of graphene sample in

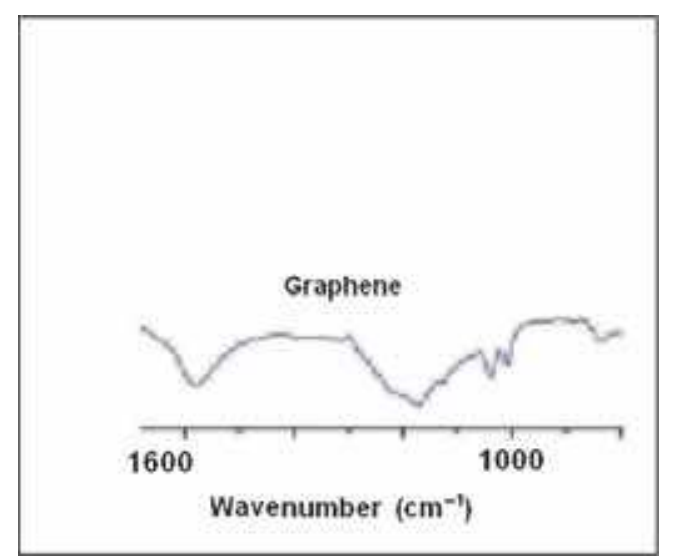

Figure 6. The FTIR spectra of graphene sample in a close window of wavenumbers $\left(800-1600 \mathrm{~cm}^{-1}\right)$.

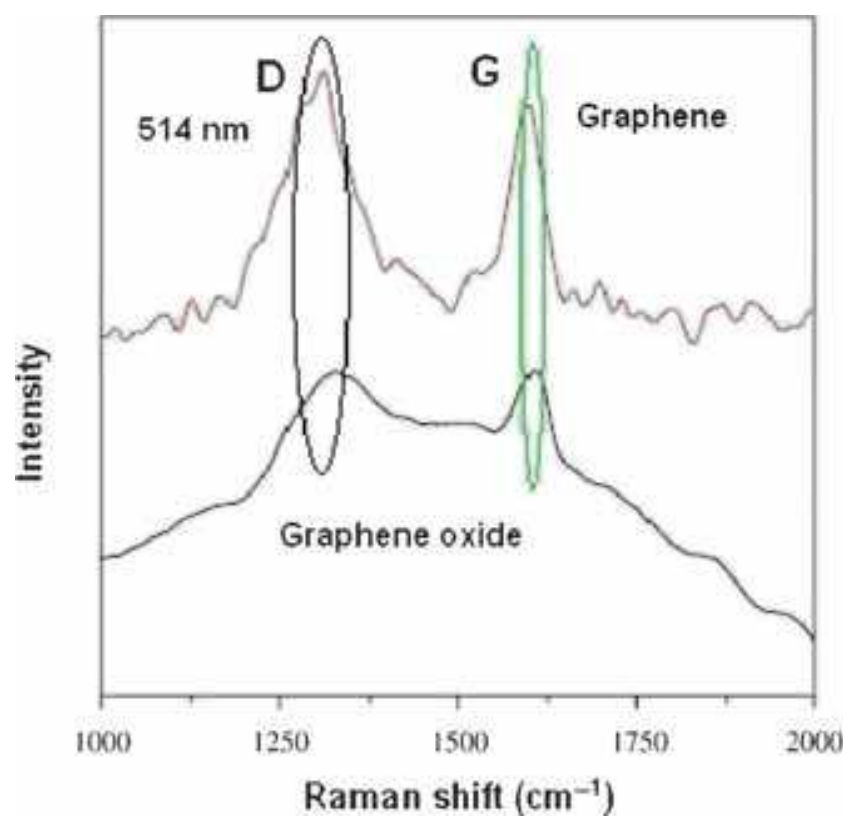

Figure 7. The Raman spectra of graphene oxide and graphene in the region $1000-2000 \mathrm{~cm}^{-1}$, indicating the presence of $\mathrm{D}$ and $\mathrm{G}$ bands.

a close window $\left(800-1600 \mathrm{~cm}^{-1}\right)$. The peaks at 1175 , 1126 and $1040 \mathrm{~cm}^{-1}$ (two S-O and one S-phenyl) confirm the presence of a sulphonic acid group, and the peaks at $1007 \mathrm{~cm}^{-1}$ (C-H in-plane bending) and $830 \mathrm{~cm}^{-1}$ (out-ofplane hydrogen wagging) are characteristic vibrations of a $p$-disubstituted phenyl group [18].

Figure 7 shows the Raman spectra of GO and graphene in the region $1000-2000 \mathrm{~cm}^{-1}$, indicating the presence of two prominent peaks at 1350 and $1620 \mathrm{~cm}^{-1}$, which corresponds to $\mathrm{D}$ and $\mathrm{G}$ bands, respectively [19]. It is obviously noticed that these two bands are broader in GO than those in graphene, corresponding to higher disorder in GO. The sharpening of the G band and an increase in the peak intensity in graphene are due to the restoring $\mathrm{sp}^{2}$ domains and the reestablished $\mathrm{sp}^{2}$ network after the complete reduction. The D peak intensity is not related to the 
number of layers present in the graphene sample, but corresponds to the disorder. Occurrence of $D$ and $G$ peaks reveal the presence of defects in our synthesized graphene sample, as D peak is totally absent in defect free singlelayer pristine graphene $[20,21]$. The $\mathrm{G}$ peak corresponds to the high-frequency $E_{2 g}$ phonon at the Brillouin zone centre $\Gamma$ [22], while the D peak corresponds to the breathing modes of six-atom rings. Figure 8 illustrates the comparative Raman spectra of the as-synthesized graphene sample with bulk graphite at $514 \mathrm{~nm}$ in the full range of 1000$3000 \mathrm{~cm}^{-1}$. The two most important features in spectra are the $G$ peak at $\sim 1620 \mathrm{~cm}^{-1}$ and a band at $\sim 2700 \mathrm{~cm}^{-1}$, which is named as $\mathrm{G}^{\prime}$. It is the second most prominent peak always observed in graphite samples [23]. The $\mathrm{G}^{\prime}$ band has no connection with the $\mathrm{G}$ peak, but is related to the second order of zone-boundary phonons. Therefore, for simplicity, we refer to the $\mathrm{G}^{\prime}$ peak as $2 \mathrm{D}$. As seen from the figure, there is a significant change in shape and intensity of the $2 \mathrm{D}$ peak of graphene compared with bulk graphite. Graphene exhibits a single 2D peak, while graphite can exhibit double structure of the 2D peak, which can be explained by the concept of Kohn anomalies [24] as described by Ferrari and his group. It is important to mention here that although Raman spectroscopy can be applied to graphene samples growth on different substrates [25] to identify the number of layers, it is very difficult to detect number of layers in case of chemically synthesized grapheme due to the presence of large numbers of defects.

Thermal stability of both GO and graphene were examined by TGA analysis and the results of the TGA analysis are shown in figure 9. GO shows small mass decrease from room temperature to $150^{\circ} \mathrm{C}$ and significant decrease from 150 to $200^{\circ} \mathrm{C}$. The mass of GO further decreased slowly up to $600^{\circ} \mathrm{C}$. The major mass reduction at $\sim 200^{\circ} \mathrm{C}$ was caused by pyrolysis of the oxygen-containing functional groups,

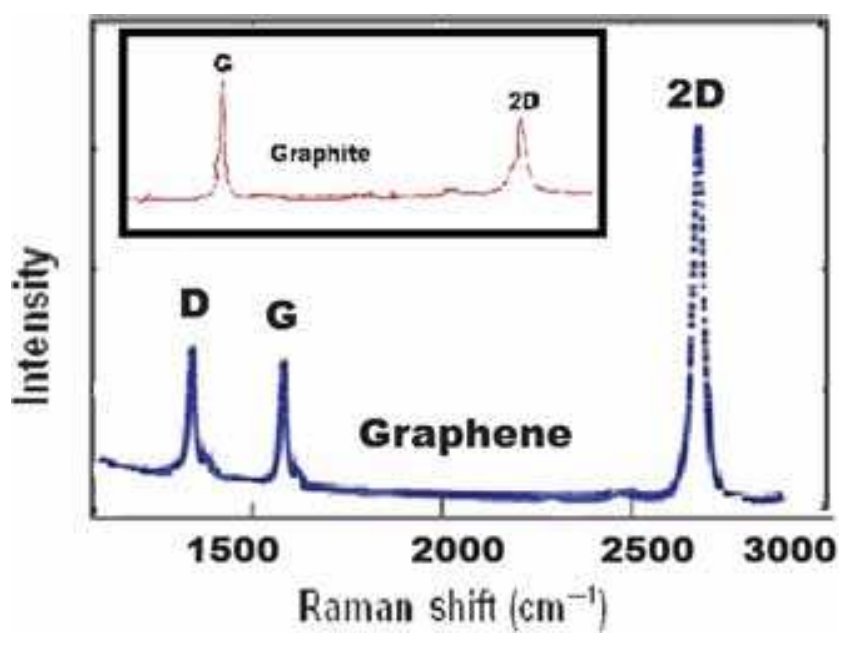

Figure 8. The Raman spectra of as-synthesized graphene sample in the full range of $1000-3000 \mathrm{~cm}^{-1}$, indicating the presence of $\mathrm{D}, \mathrm{G}$ and $2 \mathrm{D}$ peaks. The inset shows the Raman spectra of bulk graphite. generating $\mathrm{CO}, \mathrm{CO}_{2}$ and stream [26]. On the other hand, reduced graphene showed an enhanced thermal stability because of the removal of oxygen-containing functional groups by three reducing agents [27].

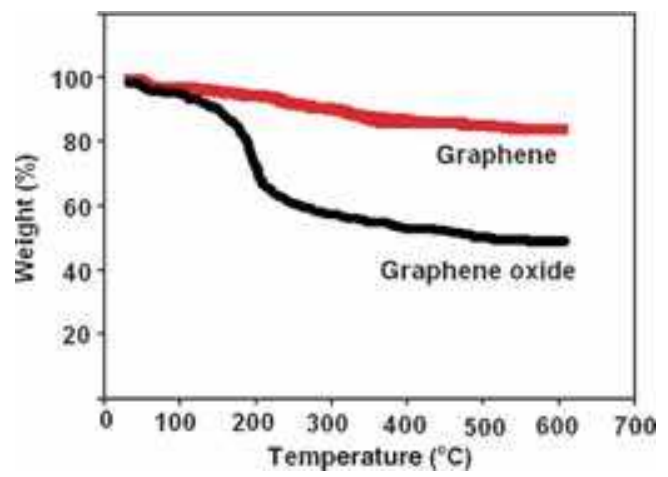

Figure 9. The results obtained from the TGA analysis of graphene oxide and graphene.

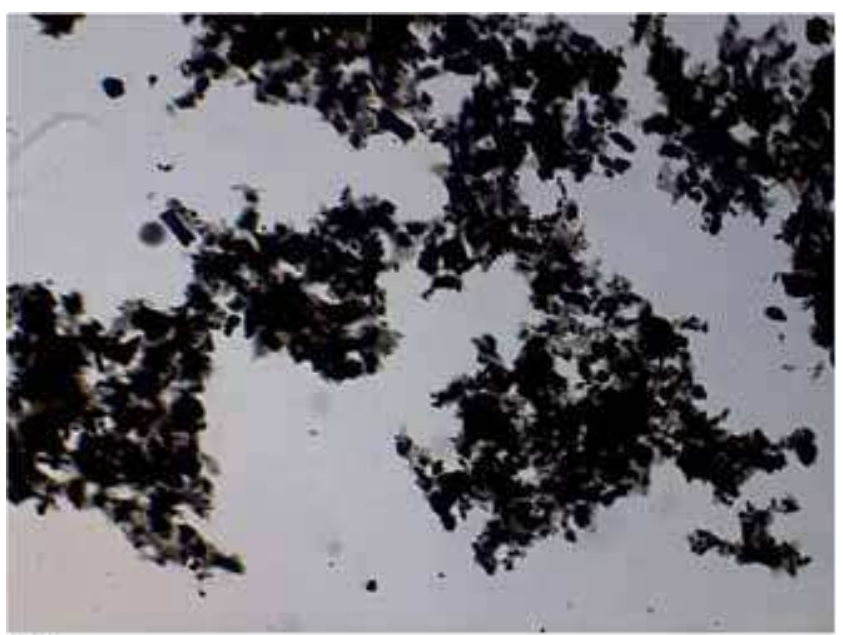

(a)

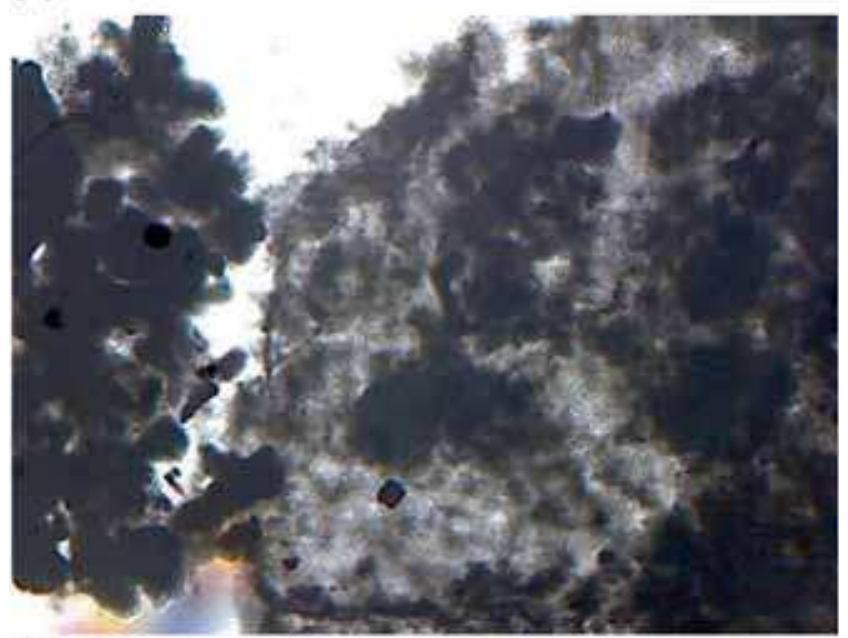

(b)

Figure 10. The optical microscopic images of as-synthesized graphene at (a) lower and (b) higher resolutions. 
Table 1. The comparison of yield and purity of water-soluble graphene synthesized by chemical methods.

\begin{tabular}{|c|c|c|c|}
\hline Synthetic protocol/reducing agents & Name of the research group with reference & Yield $(\%)$ & Purity $(\%)$ \\
\hline Chemical synthesis/sodium borohydride, hydrazine hydrate & Ankamwar and Surti [29] & $80-85$ & $90-95$ \\
\hline Chemical synthesis/sodium borohydride, hydrazine hydrate & $\mathrm{Si}$ and Samulski [30] & $85-90$ & $95-99$ \\
\hline $\begin{array}{l}\text { Chemical synthesis/sodium borohydride, hydrazine hydrate } \\
\text { and dimethyl hydrazine }\end{array}$ & Sur et al (communicated 2014) & $>90$ & $>99.99$ \\
\hline
\end{tabular}

Figure 10 illustrates the optical microscopic images of the as-synthesized graphene both at lower and higher resolution. The optical microscopic images reveal flakes like structures of the samples.

\subsection{Verification of the stability of aqueous dispersion of graphene}

The stability of the synthesized aqueous dispersion of graphene was verified by zeta-potential measurement. The magnitude of the zeta potential value gives an indication of the potential stability of the colloidal system. If all the particles in suspension have a large negative or positive zeta potential then they will tend to repel each other and there will be no tendency for the particles to aggregate. The zeta potential was calculated from the electrophoretic mobility using the Smoluchowski equation with the help of commercial software using a dynamic light-scattering (DLS) set-up. Smoluchowski equation can be expressed as $\mu=\zeta \xi / \eta$, where $\mu$ is the electrophoretic mobility, $\xi$ the electrical permittivity of the liquid, $\eta$ the viscosity and $\zeta$ the zeta potential.

We find negative zeta potentials of $55-60 \mathrm{mV}$ for the graphene dispersion. These ranges of zeta-potentials are ideal for highly stable colloidal sols. The aqueous dispersion of our synthesized graphene sheets shows good stability as evident from the zeta potential value mentioned above. The high stability of such dispersion can be justified by the explanation given by Li et al [28], which shows the electrostatic stabilization of graphene sheets because of the presence of negatively charged $-\mathrm{SO}_{3}^{-}$units providing electrostatic repulsion to keep the carbon sheets separated during reduction.

There is no sign of coagulation or agglomeration of the aqueous dispersion of graphene sheets even after 3 months. In contrast, if GO is reduced with hydrazine hydrate or dimethylhydrazine directly under similar conditions avoiding steps 1 and 2, the resulting product precipitates in water. This is due to the removal of oxygen functionality in the final product, which causes aggregation to facilitate precipitation in water. However, dispersability in water can be improved by introduction of hydrophilic group via prereduction (step 1) and sulphonation (step 2) to yield lightly sulphonated aqueous dispersion of graphene nanosheets. The presence of negatively charged $\mathrm{SO}_{3}^{-}$units, which can cause electrostatic repulsion to keep the carbon sheets separated during the reduction step. The resulting graphene remains soluble in water without any aggregation. Table 1 illustrates the comparison of yield and purity of water-soluble graphene synthesized by chemical methods. Compared with the graphene sample prepared by other groups $[29,30]$, the water-soluble graphene is extremely pure $(>99.99 \%)$ with high yield $(>90 \%)$ as demonstrated in table 1 . This shows the effectiveness and novelty of the synthetic protocol used by our group to synthesize water-soluble graphene.

At present, we are involved in the synthesis of graphene from GO using gamma radiation as well as from the biomolecules present in the plant and fruit extracts, which will be communicated later.

\section{Conclusions}

A simple synthetic protocol for the preparation of aqueous dispersion of graphene is described here. The synthesized graphene sheets were characterized by SEM, TEM, UVVis spectroscopy, FTIR and Raman spectroscopy, TGA and optical microscopy. The stability of aqueous dispersions of graphene was confirmed through zeta potential measurements and the negative zeta potentials of $55-60 \mathrm{mV}$ were obtained, indicating the high stability of aqueous graphene dispersions. The synthesized graphene nanosheets were extremely pure (>99.99\%) and the yield was high $(>90 \%)$.

\section{Acknowledgements}

UKS would like to acknowledge financial support from the projects funded by the UGC, New Delhi (Grant no. PSW045/13-14-ERO) and UGC-DAE CSR, Kolkata Centre, Collaborative Research Schemes (UGC-DAE-CSR-KC/ CRS/13/RC11/0984/0988). UKS would also like to thank INSA, New Delhi (SP/VF-9/2014-15/273 1st April, 2014), for INSA visiting Scientist Fellowship for 2014-2015.

\section{References}

[1] Kroto H W, Heath J R, O'Brien S C, Curl R F and Smalley R E 1985 Nature 318162

[2] Iijima S 1991 Nature 35456

[3] Novoselov K S, Geim A K, Morozov S V, Jiang D, Zhang Y, Dubonos S, Grigorieva I V and Firsov A A 2004 Science 306 666

[4] Geim A K and Novoselov K S 2007 Nat. Mater. 6183

[5] Rao C N R, Sood A K, Subrahmanyam K S and Govindaraj A 2009 Angew. Chem. Int. Ed. 487752 
[6] Tung V C, Allen M J, Yang Y and Kaner R B 2009 Nat. Nanotechnol. 425

[7] Choucair M, Thordarson P and Stride J A 2009 Nat. Nanotechnol. 430

[8] Berger C, Song Z M, Li X B, Wu X S, Brown N, Naud C, Mayou D, Li T B, Hass J, Marchenkov A N, Conrad E H, First P N and de Heer W A 2006 Science 312 1191

[9] Sutter P W, Flege J-I and Sutter E A 2008 Nat. Mater. 7 406

[10] Yang X, Dou X, Rouhanipour A, Zhi L, Rader H J and Mullen K 2008 J. Am. Chem. Soc. 1304216

[11] Park S and Ruoff R S 2009 Nat. Nanotechnol. 4217

[12] Ruoff R S 2008 Nat. Nanotechnol. 310

[13] Hummers W S and Offeman R E 1958 J. Am. Chem. Soc. 80 1339

[14] Kovtyukhova N I, Ollivier P J, Martin B R, Mallouk T E, Chizhik S A, Buzaneva E V and Gorchinskiy A D 1999 Chem. Mater. 11771

[15] Thakur S and Karak N 2012 Carbon 505331

[16] Xu Y X, Bai H, Lu G W, Li C and Shi G Q 2008 J. Am. Chem. Soc. 1305856

[17] Stankovich S, Piner R D, Nguyen S T and Ruoff R S 2006 Carbon 443342
[18] Colthup N B, Daly L H and Wiberley S E 1990 Introduction to infrared and Raman spectroscopy (London: Academic Press) 3rd ed

[19] Kudin K N, Ozbas B, Schniepp H C, Prud'homme R K, Aksay I A and Car R 2008 Nano Lett. 836

[20] Ferrari A C and Basko D M 2013 Nat. Nanotechnol. 8235

[21] Ferrari A C et al 2006 Phys. Rev. Lett. 97187401

[22] Nemanich R J, Lucovsky G and Solin S A 1977 Solid State Commun. 23117

[23] Vidano R P, Fishbach D B, Willis L J and Loehr T M 1981 Solid State Commun. 39341

[24] Piscanec S, Lazzeri M, Mauri F, Ferrari A C and Robertson J 2004 Phys. Rev. Lett. 93185503

[25] Das A, Chakraborty B and Sood A K 2008 Bull. Mater. Sci. 31 579

[26] Lerf A, He H, Forster M and Klinowski J 1998 J. Phys. Chem. B 1024477

[27] Stankovich S, Dikin D A, Piner R D, Kohlhaas K A, Kleinhammes A, Jia Y, Wu Y, Nguyen S T and Ruoff R S 2007 Carbon 451558

[28] Li D, Muller M B, Gilje S, Kaner R B and Wallace G G 2008 Nat. Nano 3101

[29] Ankamwar B and Surti F 2012 Chem. Sci. Trans. 1500

[30] Si Y and Samulski E T 2008 Nano Lett. 81679 